\title{
AA $\beta$ public key cryptosystem - a comparative analysis against RSA and ECC
}

\begin{abstract}
This paper aims to provide a comparative analysis between probabilistic and deterministic security models. We provide a benchmark by practically implementing and comparing three ciphers - AA6, RSA and ECC. This paper provides the algorithms to implement these ciphers as well as highlights the operating time and performance for varying key sizes of $128 / 1024 / 3072,160 / 2048 / 6144$ and 192/4096/12288 bits. We target our implementation to justify if the probabilistic model - AA 6 , can perform equivalently against deterministic models so as to be considered to be used in practical scenarios today.
\end{abstract}

Keyword: AA6 cryptosystem; Cipher implementation; Comparative analysis; Probabilistic cipher; Public-key cryptosystem 